\title{
Quantification of Blonanserin in Human Plasma Using Liquid Chromatography- Electrospray lonization-Tandem Mass Spectrophotometry-Application to Pharmacokinetic Study
}

\author{
Mondal Prasenjit' ${ }^{1}$, Shobharani Satla ${ }^{2}$, Ramakrishna Raparla ${ }^{3}$ \\ 'Department of Pharmaceutical Analysis, Vaageswari College of Pharmacy, Karimnagar, Telangana, INDIA. \\ 2Centre for Pharmaceutical science, JNTU Hyderabad, Kukatpally, Hyderabad, INDIA. \\ ${ }^{3}$ Department of Pharmacy, Vaageswari Institute of Pharmaceutical sciences, Karimnagar, Telangana, INDIA.
}

\begin{abstract}
Background: A novel LC-MS/MS method was developed for the estimation of blonanserin (BLN) in spiked human plasma. Methods: Liquid-liquid extraction (LLE) technique was adopted for the extraction of BLN from human plasma and chromatographic separation was performed on a waters symmetry shield, C18 $(4.6 \mathrm{~mm}$ id $\times 50 \mathrm{~mm})$ analytical column using $7 \mathrm{Mm}$ ammonium formate and acetonitrile $(30: 70) \mathrm{v} / \mathrm{v}$ as mobile phase. Positive ion mode was selected to obtain the product ion $\mathrm{m} / \mathrm{z} 367.24 \rightarrow 296.19$ for BLN and $\mathrm{m} / \mathrm{z} 326.8 \rightarrow 269.07$ for clozapine as internal standard (IS) Results: Calibration curve was linear over the range of 0.01 to $5 \mathrm{ng} / \mathrm{ml}$. Developed method was satisfactory validated as per US-FDA guidelines for the bioanalytical study because it exhibits excellent intra and interday accuracy with \% nominal $90 \rightarrow 98.4 \%$, precision $\% \mathrm{CV} \leq 2 \%$ in all quality control levels, shows acceptable \% extraction recovery $(95.15 \% \rightarrow 97.04 \%$ ), demonstrated excellent matrix and analyte selectivity ( $\%$ interference $=0$ ), matrix effect (matrix factor 0.981 at LQC and 1.02 at $\mathrm{HQC}$ level) and satisfactory stability study results in all types (\% nominal $93.91 \% \rightarrow 99.58 \%$ ). Along with pharmacokinetic study rabbit plasma samples also analysed for
\end{abstract}

one batch of accuracy, precision, matrix effect and rationalized the suitability of the developed method in other preclinical sample species Conclusion: Present method was successfully optimised, validated and applied favorable for the pharmacokinetic study of marketed formulation in rabbit blood samples in single oral human equivalent dose. The applicability of the developed method undoubtedly can further extend during preclinical and clinical trials.

Key words: Blonanserin, LCMS/MS, Validation, Plasma, Pharmacokinetic. Correspondence :

Prasenjit Mondal,

Department of Pharmaceutical Analysis, Vaageswari College of Pharmacy, Karimnagar, INDIA.

Phone no: +918099879341

E-mail: Prasenjit_mondal31@rediffmail.com

DOI: $10.5530 /$ jyp.2016.4.17

\section{INTRODUCTION}

Schizophrenia is devastating psychiatric disorders characterized by positive, negative, affective and cognitive syndrome ${ }^{1}$ which also leads to development of other co-morbid diseases. ${ }^{2}$ It disrupts mental and social functioning, destroys the lives of a patients as well as their family, friends. A number of antipsychotics have been explored over last forty years but the outcomes for patients still unsatisfactory ${ }^{3}$ Blonanserin (BLN) a newer atypical antipsychotic belongs to a series of 4 phenyl2-(1-piperazenyl) pyridines, chemical structure shown in Figure 1, which acts as an antagonist at dopamine $\mathrm{D}_{2}, \mathrm{D}_{3}$ and $5 \mathrm{HT}_{2 \mathrm{~A}}$ receptors. ${ }^{4}$ With many second generation antipsychotics BLN is significantly more efficacious in the treatment of schizophrenia. ${ }^{5}$ BLN is still not official in any pharmacopoeia and based on its wide acceptability throughout world, the detail analytical profile and method of quantification of it in human plasma must be reliable, economic and highly validated. Easy, reliable, economic and accurate method of drug analysis is always well accepted, because this finally minimized the cost of dosage from ${ }^{6}$. Keeping the focus on worldwide huge acceptability of BLN and availability of its very limited analytical methods in plasma, present work has been planned. A details review of literature reveals that there is an availability of very few methods for the determination of BLN in human plasma and urine. Four important factors must have to consider for plasma analysis of drug during the development of new method are reasonable elution time, an appropriate LLOQ, low cost, simple and efficient sample processing. In a single HPLC method ${ }^{7}$ where the run time of each analytes were 6 minutes which is too long. The reported UPLC/MS method ${ }^{8}$ utilized solid phase cartridges for extraction, which is generally costly and consider complex because of further recovery of analyte from the cartridges is require, where the chance of sample loss is high. Authors also followed complex gradient elution system for the elution of analyte. One reported GS-MS method ${ }^{9}$ uses also solid phase extraction which is considered costly because of its cartridges, and complex because of analyte recovery and selection of proper washing solution, compared to simple LLE (liquid-liquid extraction) and reported method exhibits longer analysis time. Another reported method for the determination of BLN in urine ${ }^{10}$ found less sensitive and shows retention time 4.2 minutes and 5 minutes run time, which generally consider too long in case of LC-MS analysis. One reported method ${ }^{11}$ used protein precipitation for the extraction of sample form the matrix shows poor peak shape and peak intensity, also exhibits long run time and retention time for the analytes. Therefore these assay methods have several significant disadvantages such as low sensitivity, complexity in the method, larger volume of sample requirement, time consuming and expensive which limits there omnipresent acceptability in clinical trial and other studies. Herein a rapid, sensitive fast and easy method was developed with the use of $300 \mu \mathrm{l}$ of plasma samples. Run time was $2.5 \mathrm{~min}$ for each sample with the use of simple Liquid-liquid extraction technique which yields better assay results. For LC-MS/MS analysis, there should be a proper extraction procedure is mandatory which can yields good recovery with minimal or no matrix effect ${ }^{12}$, therefore authors established this method as a novel, and reliable then other reported methods for the determination of BLN in human plasma based on its empirical evidences shown in Figure 2 in the form of comparison chart and validated as per USFDA bioanalytical method development. ${ }^{13}$ So that this method can better applicable for the further bioequivalence and toxicological study during clinical and preclinical trials. 


\section{MATERIALS AND METHODS}

Blonanserin standard (99.84\% pure) and Clozapine (99.87\% pure) was kindly provided by Zydus Cadila healthcare Ltd, Ahmadabad, India, as a gift sample. Ammonium formate buffer was obtained from SigmaAldrich, Hyderabad, India.

\section{High Performance Liquid chromatographic operating conditions}

An isocratic elution technique was adopted with the mobile phase (7 mM ammonium formate: acetonitrile, 30:70) derived at a flow rate of $600 \mu \mathrm{l} /$ minute using waters Symmetry shield RP C-18 column with 4.6 $\mathrm{mm}$ internal diameter, $5 \mu \mathrm{m}$ particle size, $100 \mathrm{~A}^{0}$ pore size. Under these condition retention time of BLN was $1.00 \pm 0.3 \mathrm{~min}$ and $1.10 \pm 0.3 \mathrm{~min}$ for internal standard clozapine Column effluent was introduced in to mass chamber and temperature of the auto sampler was maintained $4^{\circ} \mathrm{C}$. Run time was up to 2.5 minutes.

\section{Mass spectrometry operating conditions}

Mass spectrometry was performed using an API-3000 triple quadrupole mass spectrometer (AB SCIEX, Foster city, CA/concord, Ontario, Canada) was equipped with an electrospray ionisation source (ESI), operating in the positive ion mode at $800^{\circ} \mathrm{C}$ desolvation temperature. The ion source parameters for examples capillary voltages $3.30 \mathrm{kV}$, cone voltages $35 \mathrm{kV}$, source temperature $400^{\circ} \mathrm{C}$, desolvation gas flow $800 \mathrm{~L} / \mathrm{h}$ and other parameters were also set for the analysis BLN and internal standard were summarised in Table 1. Detection of the ions were carried out in multiple reaction monitoring by monitoring the transition pairs of $\mathrm{m} / \mathrm{z}$ 367.24-296.19 and m/z 326.8-269.07 for internal standard was shown in Figure 3 and 4.

\section{Preparation of calibration curve and quality control samples}

The calibration curve and quality control samples of BLN were prepared from the aqueous dilutions separately. Stock solution $(10000 \mathrm{ng} / \mathrm{ml})$ was prepared by dissolving $1 \mathrm{mg}$ of BLN in $1 \mathrm{ml}$ of acetonitrile and make $100 \mathrm{ml}$ with diluent (mobile phase). From the stock solution aqueous calibration curve dilutions were made to obtain the concentrations 100 , $50,15,10,5,2,1,0.2 \mathrm{ng} / \mathrm{ml}$. Finally plasma samples were prepared by diluting $0.25 \mathrm{ml}$ aliquot form different aqueous dilutions. $0.75 \mathrm{ml}$ of plasma matrix were added to each tubes and volumes were made up to $5 \mathrm{ml}$ with diluent to obtain the final concentration 5 to $0.01 \mathrm{ng} / \mathrm{ml}$. Quality control samples were also prepared in same way from the different aqueous QC dilutions to obtain the final concentrations at LLOQ $(0.01 \mathrm{ng} / \mathrm{ml})$, LQC $(0.25 \mathrm{ng} / \mathrm{ml})$, MQC (2.5 ng/ml), HQC (5 ng/ml), DIQC (25 ng/ml) levels.

\section{Sample preparation}

A simple liquid-liquid extraction technique was adopted for the sample preparation. The plasma samples of the analytes and internal standard for calibration curve and quality control samples were thawed at room temperature and vortexed the samples to ensure the complete mixing. $250 \mu \mathrm{l}$ of plasma sample was pipette out and placed in to $15 \mathrm{ml}$ of Stoppard tube. $25 \mu \mathrm{l}$ of internal standard from $0.25 \mu \mathrm{g} / \mathrm{ml}$ clozapine dilution was added to each Stoppard tube and vortexed except the blank plasma samples where $25 \mu \mathrm{l}$ of diluent (mobile phase) was added. $5 \mathrm{ml}$ of tetra butyl methyl ether as extracting solvent was added to each tube and shaken for 20 minutes on reciprocating shaker at $200 \mathrm{rpm}$. Samples were then centrifuged at $4000 \mathrm{rpm}$ for 10 minutes at $25^{\circ} \mathrm{C}$. The supernatant organic layer was transferred into pre labelled tubes and evaporated to dryness under nitrogen gas at $40^{\circ} \mathrm{C}$. All the samples were reconstituted with $300 \mu \mathrm{l}$ of mobile phase and transferred in to auto sampler loading vials and $10 \mu \mathrm{l}$ of sample was injected in to the LC-MS/MS system.

\section{Method validation}

The optimised method was validated as per sated guidelines of bioanalytical method development and validation of by USFDA. The developed method was validated for matrix effect, carry over effect, accuracy, precision, recovery, sensitivity and different stability parameters.

\section{Carry over effect}

This was performed to investigate the effect of carrying analytes and internal standard in different sections of the system. Six replicates of the extracted blank matrix and six replicates of the extracted high concentration of analytes at the calibration curve range (ULOQ), six extracted LLOQ concentrations of the analytes and internal standard were prepared and injected as per sequence The sequence is, first six injections was extracted LLOQ, followed by extracted ULOQ and extracted blank plasma. ULOQ and blank plasma samples were injected alternatively. Response of interfering peaks in blank samples at the retention time of the analytes must be $\leq 20 \%$ of average drug response and for internal standard must be $\leq 5 \%$ of average internal standard response in LLOQ samples.

\section{Matrix effect}

It was determined at two concentration level (LQC and HQC) in eight replicates for analyte and internal standard. One haemolytic and one lipemic plasma lots has been selected. One set of each of samples were spiked with blank matrices (haemolytic), another set of each samples (LQC and HQC) with internal standard was spiked with lipemic plasma was prepared. Six replicates of aqueous samples equivalent to final LQC and HQC concentrations were prepared by spiking the analyte with internal standard to reconstituted solution and injected individually. IS normalized matrix factor was calculated by dividing peak response area in presence of matrix ion and mean peak area response ratio in absences of matrix ion. The variability in IS-normalized factor as measured by the coefficient of variation which should be less than $15 \%$.

\section{Matrix selectivity and specificity}

Matrix selectivity was evaluated by analysing the plasma of six different lots, including one haemolytic and one lipemic plasma to investigate the interference the retention time of the analytes and internal standard. The interference at the retention times of the drugs by comparing the response in the blank plasma, against the response of LLOQ was evaluated. And the interference at the retention time of internal standard also evaluated against the response of the extracted internal standard in LLOQ sample. The response of the interfering substance will be acceptable if it is less than $20 \%$ of the mean drug response in LLOQ sample and less than $5 \%$ in case of internal standard.

\section{Analyte selectivity}

Analytes selectivity was also analyzed to investigate the internal standards interference at analytes retention time. The investigate this six replicates of matrix blank with internal standard was injected, if any area for analytes was found was compared with mean area of the analytes obtained with LLOQ concentration injected. Similarly internal standards selectivity was also investigated for this six replicates of matrix blank with drugs was in injected at ULOQ level and area of internal standard if obtained, compared with mean area of internal standard at LLOQ level.

\section{Precision and accuracy}

The intra and interday precision and accuracy were estimated using several QC samples at the level of LLOQ, LQC, MQC and HQC in six replicates and the concentrations in these level was calculated followed 
by standard deviation, \% CV for precision and \% nominal for accuracy for each replicates. The acceptance criteria for accuracy (\% nominal) is $\pm 15 \%$ and $\pm 20 \%$ only for LLOQ level and for precision (\%CV) should be within $\pm 15 \%$ and only for LLOQ sample it is $\pm 20 \%$.

\section{Linearity}

The developed method was assessed for linearity in the concentration range of 0.01-5 ng/ml. Eleven CC (calibration curve) samples has been prepared by spiking human plasma and processed. The CC has been constructed by using a regression equation with a weighing factor $1 /$ (concentration ratio $)^{2}$ of the drug to internal standard concentration to produce best fit for the concentration/response relationship. The acceptance criteria for the linearity is the $\mathrm{r}^{2}$ (coefficient of correlation) should be $\geq 0.98$.

\section{Dilution integrity}

To investigate dilution integrity of the developed method 12 sets of QC stock solution were prepared by spiking 1.5 times of the highest standard concentration. Six sets of dilution integrity samples were prepared by diluting 2 times and another six samples by 4 times dilution. These samples were analyzed and concentrations were calculated by multiplying suitable dilution factors, 2 (for two times dilution) and 4 (for four times dilution). For each dilution level at least 67\% (4 out of 6) of the QC samples should be $15 \%$ of their respective nominal.

\section{Recovery study}

This study was conducted to express the extraction efficiency of an analytical process, by comparing the peak response from extracted and non extracted samples. Six LQC, MQC and HQC samples has been prepared freshly and these samples were processed by adding internal standard and injected. Foe non extracted samples 18 blank matrix samples were spiked with six sets of each LQC, MQC and HQC with internal standard and injected. Six non extracted samples of each 3 levels were prepared by spiking $10 \mu \mathrm{l}$ of analytes and $10 \mu \mathrm{l}$ of internal standard in extracted blank plasma. Mean overall \% recovery was calculated, and $\%$ of difference should not be more than $25 \%$ between highest and lowest $\%$ recovery.

\section{Ruggedness}

To investigate the ruggedness of the developed method, one precision and accuracy batch of samples were processed and analyzed with different columns of the same make and with different reagent lots.

\section{Stability studies}

In the stability study (Bench top, wet extract, freeze thaw, autosampler, short term and long term stability) of BLN was performed using freshly prepared calibration curve samples and quality control samples were prepared at low middle, high level and analysed. Concentration response linearity data was collected and used to calculate the concentration of stability samples.

\section{Room temperature stability study}

It was performed using the stock solution prepared for at least a period of 6 hours. Fresh stock solution of analyte and internal standard were prepared. The final dilution of stock solution (stability samples) and fresh stock solution (comparison sample) was done, which is equivalent to final middle quality control analytes and internal standard. Six replicates of fresh and comparison samples were injected immediately and $\%$ of stability was calculated. It must be between $95 \%-105 \%$ and $\% \mathrm{CV}$ should be less than $10 \%$.

\section{Refrigerator stock solution stability}

To accessed this type of stability six replicates of stock solution was prepared and stored at refrigerator at $2-8^{\circ} \mathrm{C}$ for 4 days. On the day of evaluation fresh standard stock solution was prepared (comparison sample) equivalent to final MQC concentration of the analytes with final concentration of internal standard in reconstituted solution. All comparison and stability samples were injected immediately. Percentage of stability was also calculated for the analytes and internal standard and it must be between $95 \%-105 \%$ and \%CV must be less than $10 \%$.

\section{Bench top stability}

Six sets of LQC and HQC samples were collected from the deep freezer and placed unprocessed for a period of 12 hours. After that period six sets of fresh quality control samples (low, middle, high) and calibration samples were prepared. Bench top stability samples were processed analyzed along with fresh samples. Concentration was calculated from the linearity data.

\section{Autosampler stability}

To access this type stability six sets of quality control samples were prepared in LQC and HQC level and kept in autosampler for 3 days, all the stability samples were quantified against the freshly prepared spiked calibration curve and quality control samples at low middle and high level.

\section{Freeze thaw stability}

It was determined by four freeze thaw cycles. Six replicates of LQC and HQC samples were prepared and stored in deep freezer at $-70^{\circ} \mathrm{C}$. After 24 hours first six samples were withdrawn and thawed at room temperature and refreeze again. In the similar way remaining samples also withdrawn after next 12 hours followed by another 12 hours and refreeze again. All samples were processed after suitable 4 cycles. Stability samples were analyzed by quantifying along with freshly spiked calibration samples and quality control samples at low mid and high level.

\section{Wet extract stability}

To investigate wet extract stability six replicates of LQC and HQC samples were prepared, processed and kept for one day at room temperature $\left(20 \pm 5^{\circ} \mathrm{C}\right)$. After the suitable stability period the samples were injected with freshly spiked calibration curve samples and quality control samples at low middle and high level. The amount of analytes in stability samples were calculated in compared with freshly prepared samples.

\section{Short term stability}

Six sets of quality control samples at low and high level were prepared and stored at deep freezer at $-20^{\circ} \mathrm{C}$ after spiking. Three days later on the day of evaluation samples were processed along with freshly prepared quality control samples in all levels and calibration curve samples. The concentration of the stability samples were calculated in comparison to freshly prepared samples.

\section{Long term stability}

This was investigated with LQC and HQC samples kept for 30 days at $-70^{\circ} \mathrm{C}$. On the day evaluation six sets of long term quality control samples (LQC and HQC) were withdrawn and processed them with freshly prepared calibration curve and quality control samples. All the stability samples were quantified from the calibration curve data. For all stability samples mean \% nominal concentration at each quality control level must be between $85 \%$ and $115 \%$ and the precision must be $\leq 15 \%$ of the CV \%. At least $67 \%$ of the stability QC samples should be within $15 \%$ of their respective nominal values.

\section{Pharmacokinetic study}

In vivo Pharmacokinetic study was performed using $\mathrm{NZ}$ white rabbit $(\mathrm{n}=3,2 \mathrm{~kg}$ each, 2 male, male: female 2:1) model. It was performed as per local animal ethical committee guidelines. To assess the applicability of the developed method, it was carried at $0.18 \mathrm{mg} / \mathrm{kg}$ dose for BLN. The orally administered dose was human equivalent dose to particular this 


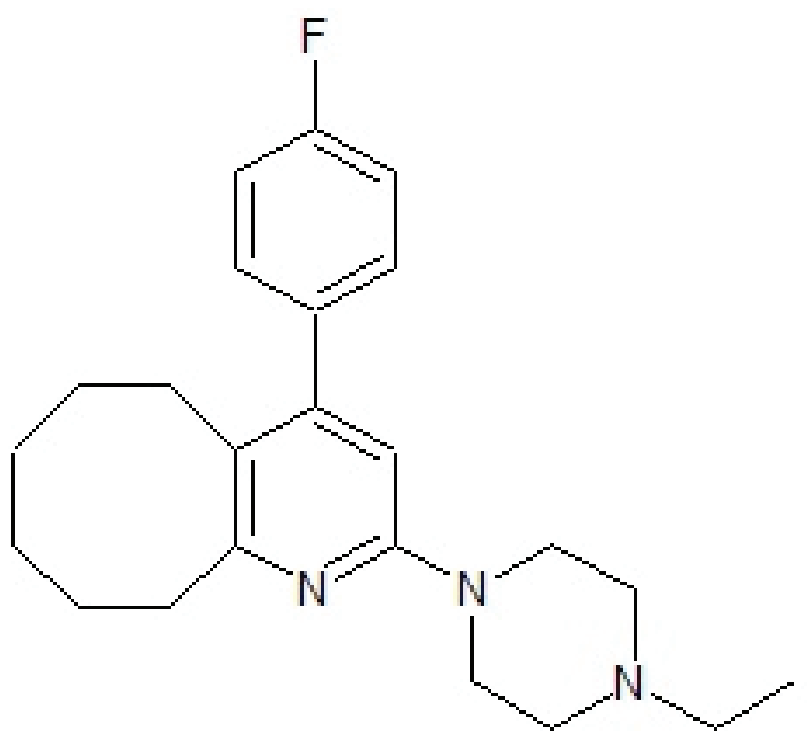

Figure 1: Chemical structure of Blonanserin.

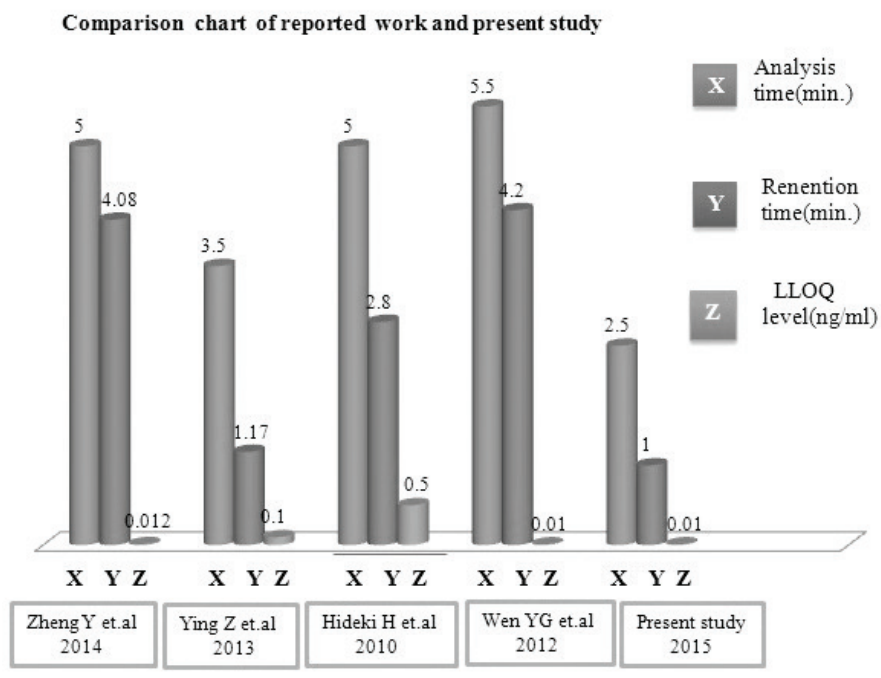

Figure 2: Comparison chart between reported and present work.

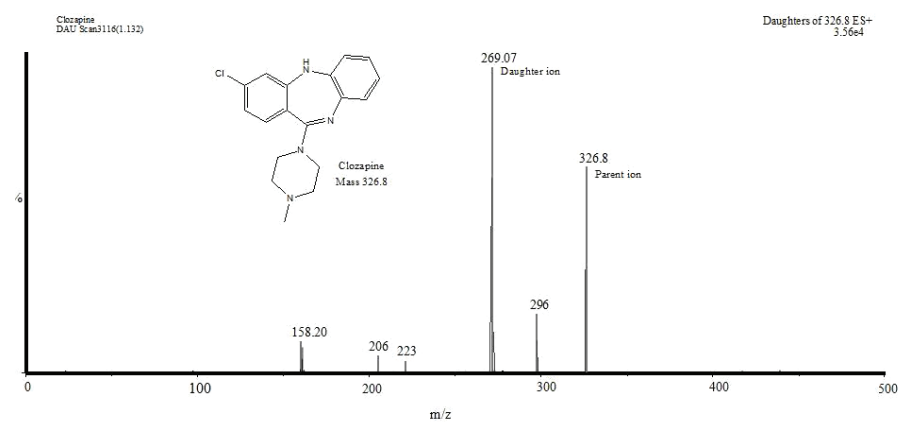

Figure 3: Product ion mass spectra of $[\mathrm{M}+\mathrm{H}]^{+}$of Blonanserin.

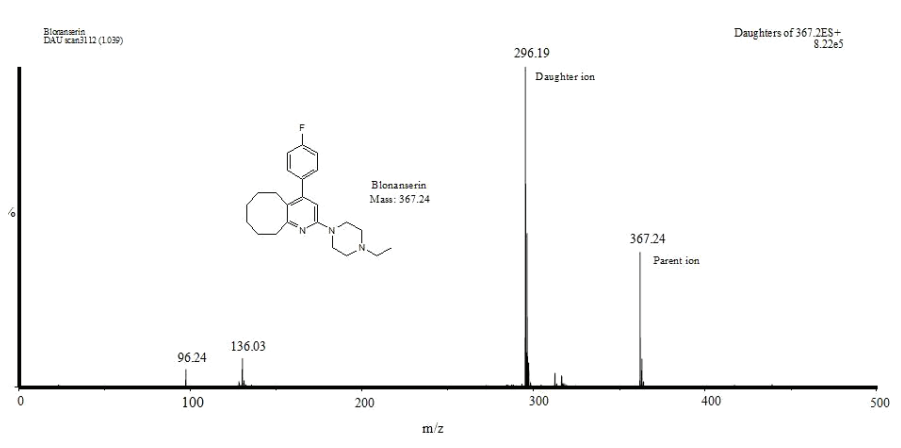

Figure 4: Product ion mass spectra of $[\mathrm{M}+\mathrm{H}]^{+}$of Clozapine.

marketed formulation, has been calculated as per US-FDA guidelines for equivalent dose calculation. ${ }^{14}$ The oral dose was administered via silicon rubber gastric intubation tube to their respective groups. Blood samples $(1.5 \mathrm{ml})$ was collected from the marginal ear vein into polypropylene tube (K2 EDTA, J.K diagnostic- Rajkot) at 0, 1, 3, 5, 8, 12, 16, 20, 24 hour post dose. Plasma was separated immediately by centrifugation $(2000 \mathrm{rpm}$, $5 \mathrm{~min}$ ) and stored at $-80^{\circ} \mathrm{C}$ until analysis. The plasma samples were spiked with internal standard and processed as per sample preparation technique described earlier. The time and plasma concentration data was analysed with a non compartment model, using PK solver. 2 a menu driven adding programme for MS excel. ${ }^{15}$ One batch of accuracy, precision and matrix effect has also performed taking rabbit plasma samples to justify that the developed method is good enough in other preclinical species plasma.

\section{RESULT AND DISCUSSION}

\section{Mass spectrometry}

Proper tuning of all mass parameters has been done in both positive and negative ion mode for the analyte and internal standard; finally positive ion mode with multiple reaction monitoring mode was selected for specificity. Optimized mass parameters have been cited in Table 1. Protonated analyte and internal standard $[\mathrm{M}+\mathrm{H}]^{+}$ion was the parent ion in the Q1 segment and use as precursor ion to obtained Q3 product ion spectra.

\section{Method development}

To develop the method several chromatographic trials were conducted using different mobile phases with different volume ratio and columns. Various combinations of acetonitrile and buffers has been used during initial trials, different types of columns like C-8, C-18 of hypersil, Zorbax, kromasil has been used. Finally waters symmetry shield, C- ${ }_{18}(4.6 \mathrm{~mm}$ id x $50 \mathrm{~mm}$ ) analytical column (waters milford, MA, USA) with the mobile phase composed of acetonitrile and $1 \%$ formic acid in the volume ratio of $(80: 20) \mathrm{v} / \mathrm{v}$ has been selected. In this optimized condition peak shape was found satisfactory for analyte and internal standard even at very low quality control sample. Initially several compounds were investigated to select a suitable internal standard, finally clozapine was selected as the retention time and other values were very selective and lack of interference with analyte. Under this optimized condition the retention time of BLN was found $1.00 \pm 0.3 \mathrm{~min}$, and for internal standard $1.10 \pm 0.3 \mathrm{~min}$ was found, shown in Figure 5.

\section{Validation}

The results of carryover test shows there was no interference at the retention time of the analyte and internal standard at ULOQ and LLOQ level. The obtained response for blank samples is 0 and calculated \% carry over is also $0 \%$ which satisfied the acceptance criteria. In the result of matrix 

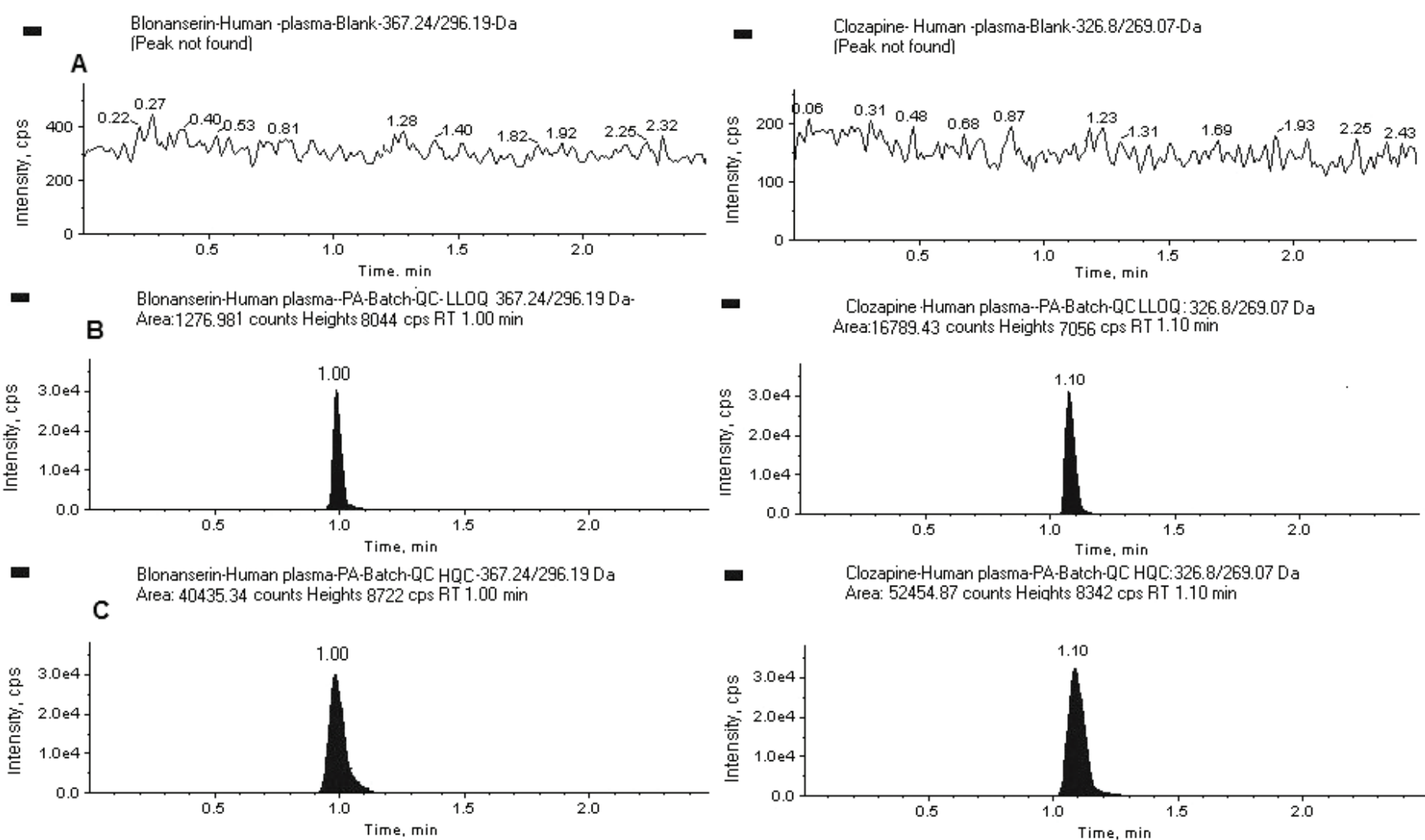

Clozapine-Human plasma-PA-Batch-QC HQC:326.8/269.07 Da

Area: 52454.87 counts Heiqhts 8342 cps RT 1.10 min

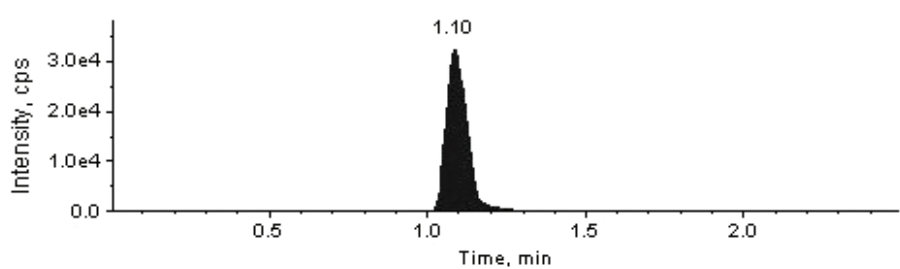

Figure 5: MRM chromatograms of Blonanserin, Blank plasma (A), LLOQ samples with internal standard [right panel] (B), HQC samples with internal Standard [right panel] (C).

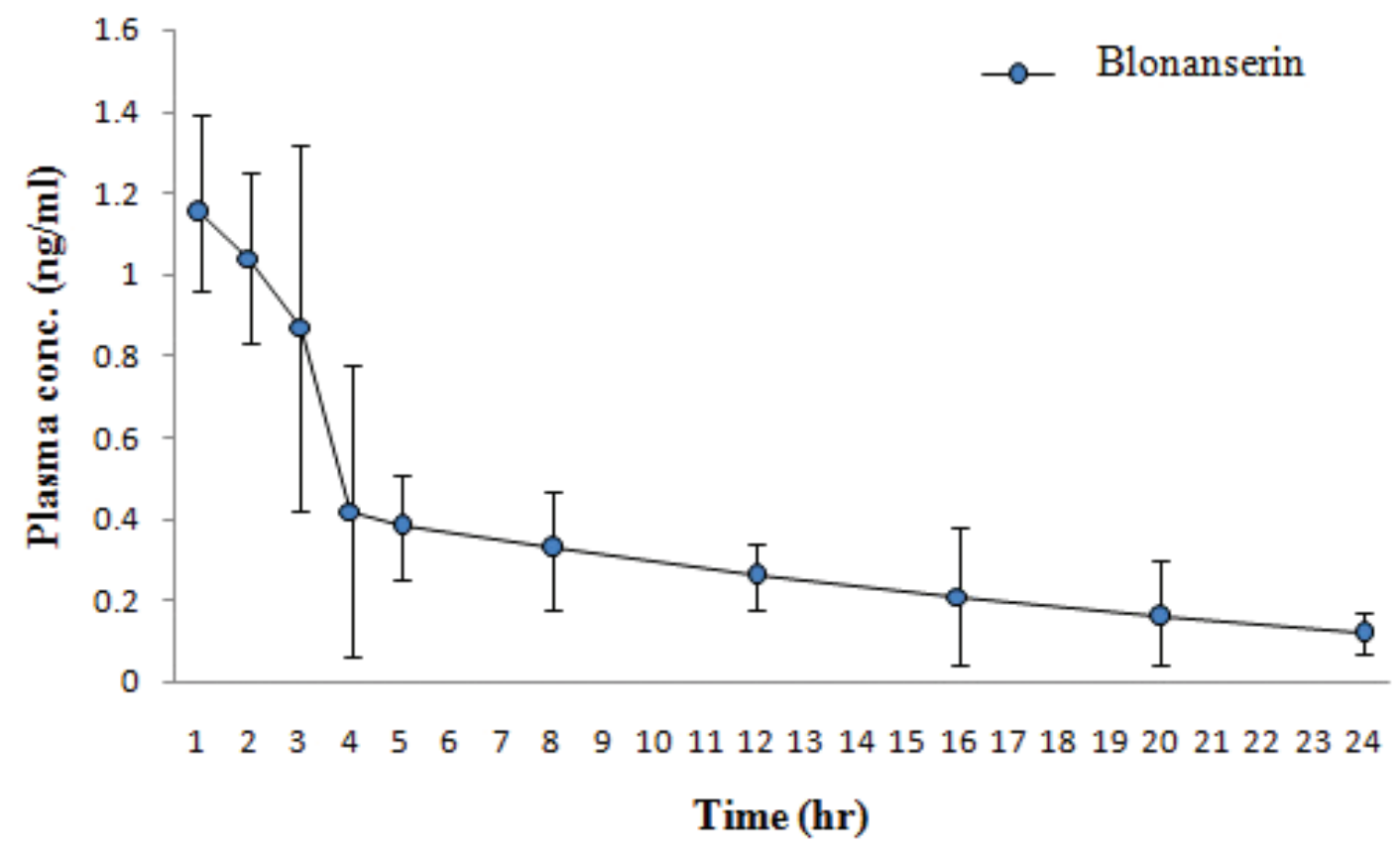

Figure 6: Mean plasma concentration -time profile of BLN after oral administration of BLN tablet human equivalent dose. 
Table 1: LC-MS/MS operating conditions (Positive ion mod

\begin{tabular}{ccc}
\hline Parameters & Blonanserin & Internal standard \\
\hline \multirow{2}{*}{ MRM } & Parent (Da) m/z 367.24 & Parent (Da) m/z 326.8 \\
\cline { 2 - 3 } & Daughter (Da) m/z 296.19 & Daughter (Da) m/z 269.07 \\
\hline Ion spray voltage & $4000 \mathrm{~V}$ & $5500 \mathrm{~V}$ \\
Source temperature & $400^{\circ} \mathrm{C}$ & $400^{\circ} \mathrm{C}$ \\
Dewll time (msec) & 200 & 200 \\
Curtain gas 1 & $20 \mathrm{psi}$ & $20 \mathrm{psi}$ \\
Declustering potential (DP) & $87 \mathrm{~V}$ & $87 \mathrm{~V}$ \\
Capillary (kv) & 3.50 & 3.50 \\
Cone (v) & 35 & 35 \\
Collision energy (CE) & 37 & 37 \\
Collision exit potential $(\mathrm{CXP})$ & 20 & 12 \\
Extractor $(\mathrm{v})$ & 5 & 5 \\
RF lens $(\mathrm{v})$ & 0 & 800 \\
Desolvation temperature $\left({ }^{\circ} \mathrm{C}\right)$ & 800 & 75 \\
Desolvation gas flow $(\mathrm{L} / \mathrm{h})$ & 75 & \\
\hline
\end{tabular}

$\mathrm{MRM}=$ multiple reaction monitoring.

Table 2: Accuracy and precision for determination of Blonanserin in human plasma

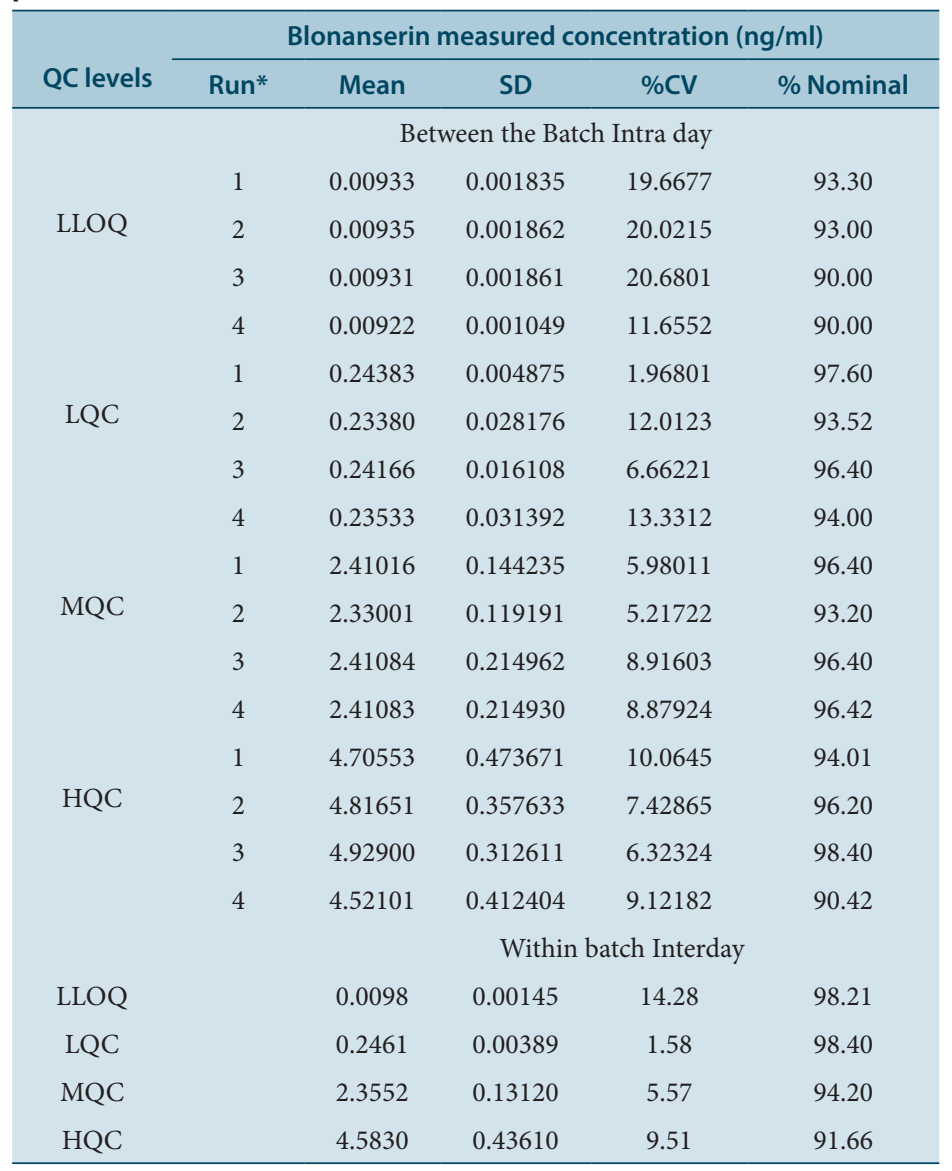

*each run includes six replicates. 
Table 3: Calibration curve concentrations and obtained percentage accuracy of individual concentration

\begin{tabular}{cccc}
\hline $\begin{array}{c}\text { Calibration curve } \\
\begin{array}{c}\text { Concentrations } \\
(\mathbf{n g} / \mathrm{ml})\end{array}\end{array}$ & $\begin{array}{c}\text { Mean of back } \\
\text { Calculated concentrations* } \\
(\mathrm{ng} / \mathrm{ml})\end{array}$ & $\begin{array}{c}\text { Standard } \\
\text { Deviation } \\
\text { (SD) }\end{array}$ & \% Accuracy \\
\hline 0.01 & 0.0106 & 0.0011 & 96.36 \\
0.02 & 0.0205 & 0.0031 & 97.15 \\
0.03 & 0.0310 & 0.0110 & 101.17 \\
0.05 & 0.0516 & 0.0030 & 102.00 \\
0.10 & 0.0866 & 0.0015 & 86.00 \\
0.25 & 0.2730 & 0.0115 & 109.20 \\
0.50 & 0.4560 & 0.0551 & 90.00 \\
0.75 & 0.6561 & 0.0310 & 87.46 \\
1.00 & 1.0530 & 0.0250 & 105.00 \\
2.50 & 2.6760 & 0.0551 & 106.80 \\
5.00 & 4.8710 & 0.0410 & 97.40 \\
\hline
\end{tabular}

${ }^{*}$ Mean of three runs of each concentration.

Table 4: Stability data of Blonanserin

\begin{tabular}{cccccc}
\hline \multirow{2}{*}{ QC levels } & & \multicolumn{4}{c}{ Blonanserin } \\
\cline { 3 - 6 } & Type of stability & Mean* & SD & \%CV & \%Nominal \\
\hline \multirow{4}{*}{ LQC } & Bench Top & 0.23633 & 0.01165 & 4.93 & 94.53 \\
& Freeze thaw & 0.24266 & 0.00943 & 3.88 & 97.06 \\
& Autosampler & 0.23701 & 0.01332 & 5.62 & 94.80 \\
& Wet extract & 0.24033 & 0.01320 & 5.49 & 96.13 \\
& Short term & 0.23834 & 0.00876 & 3.68 & 95.20 \\
& Long term & 0.23133 & 0.02324 & 10.04 & 92.52 \\
& Bench Top & 4.92833 & 0.32455 & 6.58 & 98.56 \\
& Freeze thaw & 4.77833 & 0.46799 & 9.79 & 95.56 \\
& Autosampler & 4.69566 & 0.36635 & 7.80 & 93.91 \\
& Wet extract & 4.78433 & 0.24153 & 5.04 & 95.68 \\
& Short term & 4.85633 & 0.35484 & 7.30 & 97.12 \\
& Long term & 4.95833 & 0.22578 & 4.55 & 99.16 \\
\hline
\end{tabular}

${ }^{*}$ mean of six replicates.

Table 5: Interday accuracy and precision data of rabbit blood sample

\begin{tabular}{|c|c|c|c|c|c|}
\hline \multirow[t]{2}{*}{ Analyte } & \multicolumn{5}{|c|}{ Blonanserin } \\
\hline & LLOQ & LQC & MQC-2 & MQC-1 & HQC \\
\hline Level & $3 \mathrm{ng} / \mathrm{ml}$ & $15 \mathrm{ng} / \mathrm{ml}$ & $150 \mathrm{ng} / \mathrm{ml}$ & $750 \mathrm{ng} / \mathrm{ml}$ & $1500 \mathrm{ng} / \mathrm{ml}$ \\
\hline Mean* & 2.89 & 14.69 & 146.93 & 751.8 & 1518.01 \\
\hline $\mathrm{SD}$ & 0.54 & 0.09 & 0.18 & 0.85 & 1.43 \\
\hline \%CV & 0.18 & 0.61 & 0.12 & 0.11 & 0.09 \\
\hline \%Nominal & 96.33 & 97.93 & 97.95 & 100.24 & 101.20 \\
\hline
\end{tabular}

Table 6: Pharmacokinetic parameters of BLN in rabbit plasma

\begin{tabular}{cc}
\hline Pharmacokinetic parameters & Blonanserin \\
\hline $\mathrm{C}_{0}(\mathrm{ng} / \mathrm{ml})$ & $0.81 \pm 1.65$ \\
$\left.\mathrm{C}_{\max (} \mathrm{ng} / \mathrm{ml}\right)$ & $1.45 \pm 0.46$ \\
$\mathrm{t}_{\max }$ & $2.01 \pm 1.22$ \\
$\mathrm{AUC}_{0-\infty}\left(\mathrm{h}^{*} \mathrm{ng} / \mathrm{ml}\right)$ & $10.20 \pm 3.09$ \\
$\mathrm{AUC}_{0-\mathrm{t}}\left(\mathrm{h}^{*} \mathrm{ng} / \mathrm{ml}\right)$ & $8.62 \pm 6.12$ \\
$\mathrm{t}_{1 / 2}(\mathrm{~h})$ & $9.11 \pm 6.62$ \\
$\mathrm{~V}_{\mathrm{d}}(\mathrm{L} / \mathrm{kg})$ & $9217.19 \pm 112$ \\
$\mathrm{Cl}(\mathrm{L} / \mathrm{h} / \mathrm{kg})$ & $1178.65 \pm 43.51$ \\
\hline
\end{tabular}

AUC (Area under the curve), $\mathrm{V}_{\mathrm{d}}$ (Volume of distribution), $\mathrm{Cl}$ (Clearance). 
effect, internal standard normalized matrix factor were calculated for the analyte in presence of matrix ion. No significant effect of matrix was found in eight batch includes haemolytic and lipemic plasma. Internal standard normalized factor was calculated, the \%CV of Is normalized factor was found $3.97 \%$ for LQC and $1.70 \%$ for HQC samples, satisfies acceptance criteria. Intraday within batch precision (\%CV) for LLOQ, LQC, MQC and HQC samples of BLN were found within 1\% to $14 \%$. Intraday accuracy for LLOQ, LQC, MQC and HQC was found in the range of $91 \%$ to $98 \%$. Between the batch accuracy (\% Nominal) results for the levels of LLOQ, LQC, MQC and HQC were between $90 \%$ to $98.4 \%$ and precision (\%CV) values were between $1.968 \%$ to $13.33 \%$, details were cited in Table 2 . Which indicates that the present method is highly accurate and precise. In the study of matrix selectivity no interference was found at the retention time of analyte and internal standard when compared with blank matrix against the mean response of extracted LLOQ samples and the response of interfering peaks at the retention time of analyte and internal standard was found $0 \%$ of the mean drug response. Analyte selectivity results also demonstrated that there was no interference at the retention time of analyte in presence of internal standard and also shows no interference at the retention time of internal standard in the presence of analyte. Overall matrix and analyte selectivity study demonstrated that developed method was found selective because of $0 \%$ interference. A regression equation with weighing factor,

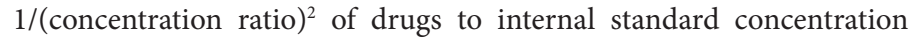
shows best fit relationship with a correlation coefficient $r^{2}=0.998$ in the concentration range of 0.01 to $5 \mathrm{ng} / \mathrm{ml}$ for BLN, with regression equation $\mathrm{Y}=0.623 \mathrm{x}$ ) confirms the linearity of the developed method. The mean back calculated concentrations for linearity with mean accuracy values has been accumulated and cited in Table 3 .In the dilution integrity study of BLN at ULOQ level was quantified. \%CV and \% nominal was found $3.179 \%$ and $98.04 \%$ at two times dilution and 0.84 and 96.08 at four times dilution in comparison with the undiluted calibration curve samples which demonstrated about the integrity of analyte in dilution. In recovery study the mean overall recovery of BLN was found $96.01 \%$ with a precision $(\% \mathrm{CV})$ range of 1.19 to 3.8 and \% difference between height and lowest \% recovery was $1.89 \%$. The mean recovery of internal standard was $94.13 \%$. Therefore the mean overall recovery results indicates that BLN and IS in LQC, MQC and HQC level were foind well satisfactory recovery and \%difference is also within acceptance level. The result of within batch precision $(\% \mathrm{CV})$ for ruggedness study was found $11.89 \%, 11.27 \%, 4.37 \%$, and $7.12 \%$. The accuracy values for ruggedness study were $95 \%, 103.22 \%, 95.29 \%, 95.68 \%$ at LLOQ, LQC, MQC and HQC levels respectively. Hence in the ruggedness study accuracy and precision values demonstrated that the developed method was found rugged on changing different column and set of reagents.

\section{Stability studies}

The results of room temperature $\left(20 \pm 5^{\circ} \mathrm{C}\right)$ stock solution stability study shows \% stability for BLN and internal standard were $98.13 \%$ and $100.79 \%$. In refrigerator stock solution stability study at $2-8^{\circ} \mathrm{C}$ for 4 days, the calculated \% of stability was found $98.19 \%$ for BLN and $96.29 \%$ for internal standard which indicates that the values were found satisfactory and within the limit and confirms the stability of the developed method. The \% nominal for Bench top stability study results were within 94 to $98 \%$ at LQC and HQC level. The autosampler stability study (72 hours of suitable stability period in autosampler) and the results of four cycles freeze thaw stability sample exhibit an excellent range of acceptability in both LQC and HQC level of Blonanserin. In wet extract stability study results \% nominal value for BLN was more than $95 \%$. The short term stability study result satisfied the acceptance limit because the calculated $\%$ nominal for BLN were more than 95\% at LQC and HQC level. The mean $\%$ nominal for long term stability study $\left(30\right.$ days at $\left.-70^{\circ} \mathrm{C}\right)$ were found 92.52 for LQC and $99.16 \%$ for HQC samples. The summary of all stability study results were demonstrated in Table 4 . In bench top, wet extract, freeze thaw, autosampler, short and long term $\left(-70^{\circ} \mathrm{C}, 30\right.$ days $)$ stability studies, stability samples were compared with freshly prepared samples and concentration was back calculated from the calibration curve samples. In all category of stability studies the mean $\%$ of nominal values were found within $94.53 \%$ to $100.79 \%$ and $\% \mathrm{CV}$ values were within $10 \%$, which strongly demonstrated about the stability of all quality control samples and stability of the developed method.

\section{In vivo pharmacokinetic study}

The pharmacokinetic parameters obtained from the study using non compartmental model, were area under the curve $\left(\mathrm{AUC}_{0}-\infty\right)$ for $\mathrm{BLN}=10.20 \pm 3.09 \mathrm{hr} . \mathrm{ng} / \mathrm{ml}$. Elimination half life $\left(\mathrm{t}^{1 / 2}\right)=9.11 \pm 6.02 \mathrm{hr}$. BLN maximum concentration in plasma $\left(\mathrm{C}_{\text {max }}\right)=1.45 \pm 0.46$ and $\mathrm{t}_{\text {max }}$ was achieved at $5 \pm 1.53 \mathrm{hr}$. Along with pharmacokinetic study rabbit plasma samples also analysed for one batch of accuracy, precision, and matrix effect, where all values are within the acceptance limit and rationalized the suitability of the developed method in other preclinical sample species. Accuracy and precision data on rabbit plasma samples were demonstrated in Table 5 and pharmacokinetic data was summarized in Table 6. The newly developed validated method was well implemented during the analysis of pharmacokinetic samples. The plasma concentration and time is smooth enough to derive all parameters (Figure 6). The clearance and elimination half life were well fitted with one compartmental model. The selectivity, sensitivity and specificity of the present method was sufficient enough for the characterization of Pharmacokinetic profile of BLN in $N Z$ white rabbit plasma.

\section{CONCLUSION}

The present method shows high throughput than the few reported LCMS/MS methods because the requirement of total analysis time is very less with less run time. A convenient liquid-liquid extraction procedure made this method more practically workable and cost effective. Empirical evidences of all validation results demonstrated that the method is highly validated and easy as all the parameters are within acceptance limit as per the US-FDA guidelines. So this present cheap attractive, easy, reliable novel method can undoubtedly highly applicable for the quantitative analysis of BLN during clinical trials, preclinical trials, forensic and toxicological study.

\section{ACKNOWLEDGEMENT}

The authors gratefully acknowledge Axis Clinicals Limited research laboratory, Hyderabad, India for providing necessary facilities for carrying out the work.

\section{CONFLICT OF INTEREST}

No potential conflict of interest was reported by the authors. 


\section{ABOUT AUTHORS}

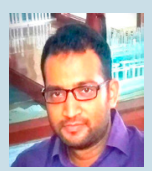

Prasenjit Mondal: Is a Associate professor at the Vaageswari college of Pharmacy, and is a doctoral student at JNTU Hyderabad. His doctoral work focused on the Bioanalytical method development and validation in human plasma using LC-MS/MS and pharmacokinetic study.

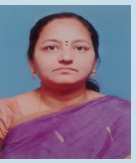

Dr. Satla Shobha Rani: Obtained her Ph.D degree from Kakatiya University, Warangal. Currently she is positioned as associate professor department of Pharmaceutical analysis, CPS, JNTU Hyderabad. She has projects from AICTE and recipients of UGC project on synthesis and pharmacological evaluation of new heterocyclic compounds. Has experience in the area of synthesis and method development and validation of new drugs using HPLC, LC-MS/MS.

\section{REFERENCES}

1. Tenjin T, Miyamoto S, Ninomiya Y, Kitajima R, Ogino S, Nobumi M, et al. Profile of blonanserin for the treatment of schizophrenia. Neuropsychiatr Dis Treat. 2013;9:587-94.

2. Stephen HS, Stephen WN, Cleveland GS. Schizophrenia: A Review. Am Fam Physician. 2007;75(12):1821-29.

3. Miyamoto S, Miyake N, Jarskog LF, Fleischhacker WW, Lieberman JA. Pharmacological treatment of schizophrenia:a critical review of the pharmacology and clinical effects of current and future therapeutic agents. Mol Psychiatry. 2012;17(2):1206-27.

4. Une T and Kurumiya S. Pharmacological profile of Blonanserin. Jpn J Clin Psychopharm. 2007;10(7):1263-72.

5. Kishi T, Matsuda Y, Nakamura H, Iwata N. Blonanserin for schizophrenia Systematic review and meta-analysis of double-blind, randomized, controlled trials. J Psychiatr Res. 2013;47(2):149-54.

6. Mondal P, Shobharani S, Ramakrishna R. Novel stability indicating validated RPHPLC method for simultaneous quantification of Artemether and Lumefantrine in Bluk and Tablet. Curr Pharm Anal. 2014;10(4):271-8.

7. Matsuda M, Sakashita M, Yamaguchi T, Fujii T. Determination of a novel antipsychotic agent AD-5423 and its metabolites in plasma by high-performance liquid chromatography with fluorescence detector. J Pharm Biomed Anal. 1997; 15(9):1449-56

8. Ying Z, Ming L, Jiang J, Wang H, Hu, P. Simultaneous determination of blonanserin and its four metabolites in human plasma using ultra-performance liquid chromatography-tandem mass spectrometry. J Chromatogr B. 2013;15(903):59-66.
9. Hideki H, Iwai M, OgawaT, MizutaniY, Ishii A, Suzuki O, Seno H. Simple analysis of blonanserin, a novel antipsychotic agent, in human plasma by GC- MS Foreign Toxicol. 2010;28(2):105-9.

10. Wena YG, Nia XJ, Zhanga M, Liua X Shang DW. Simultaneous determination of blonanserin and its metabolite in human plasma and urine by liquid chromatography- tandem mass spectrometry: Application to a pharmacokinetic study. J Chromatogr B. 2012;15(903):46-52.

11. Zheng $Y$, Hu X, Liu J, Wu G, Zhou H, Zhu M, et al. Simple and sensitive analysis of blonanserin and blonanserin $C$ in human plasma by liquid chromatography tandem mass spectrometry and its application. J Anal Methods Chem. 2014;2014(2014):1-7

12. Narapusetti A, Bethanabhatla SS, Sockalingam A, Rao PN. LC-MS/MS assay for Acetazolamide, A Carbonic Anhydrase Inhibitor in Human Plasma and its Clinical Application. J Young Pharm. 2015;7(4):438-45. http://dx.doi. org/10.1155/2014/629343

13. US Food and Drug Administration, Guidance for Industry (2013) Bioanalytical Method Validation. http://www.fda.gov/downloads/Drugs/GuidanceCompliance RegulatoryInformation/Guidances/UCM368107.pdf. Accessed 06 Jan 2015

14. US-FDA guidelines for equivalent dose calculation

15. http://www.fda.gov/downloads/Drugs/.../Guidances/UCM078932.pdf. Accessed 05 Jan 2015.

16. Zhang Y, Huo M, Zhou J, Xie S. PK Solver: An add-in program for pharmacokinetic and pharmacodynamic data analysis in Microsoft Excel. Comput Meth Prog Bio. 2010;99(3):306-14 